Elskamp, F.; Kruggel-Emden, H. ; Hennig, M.; Teipel, U.

\title{
A strategy to determine DEM parameters for spherical and non-spherical particles
}

Journal article | Accepted manuscript (Postprint)

This version is available at https://doi.org/10.14279/depositonce-8315

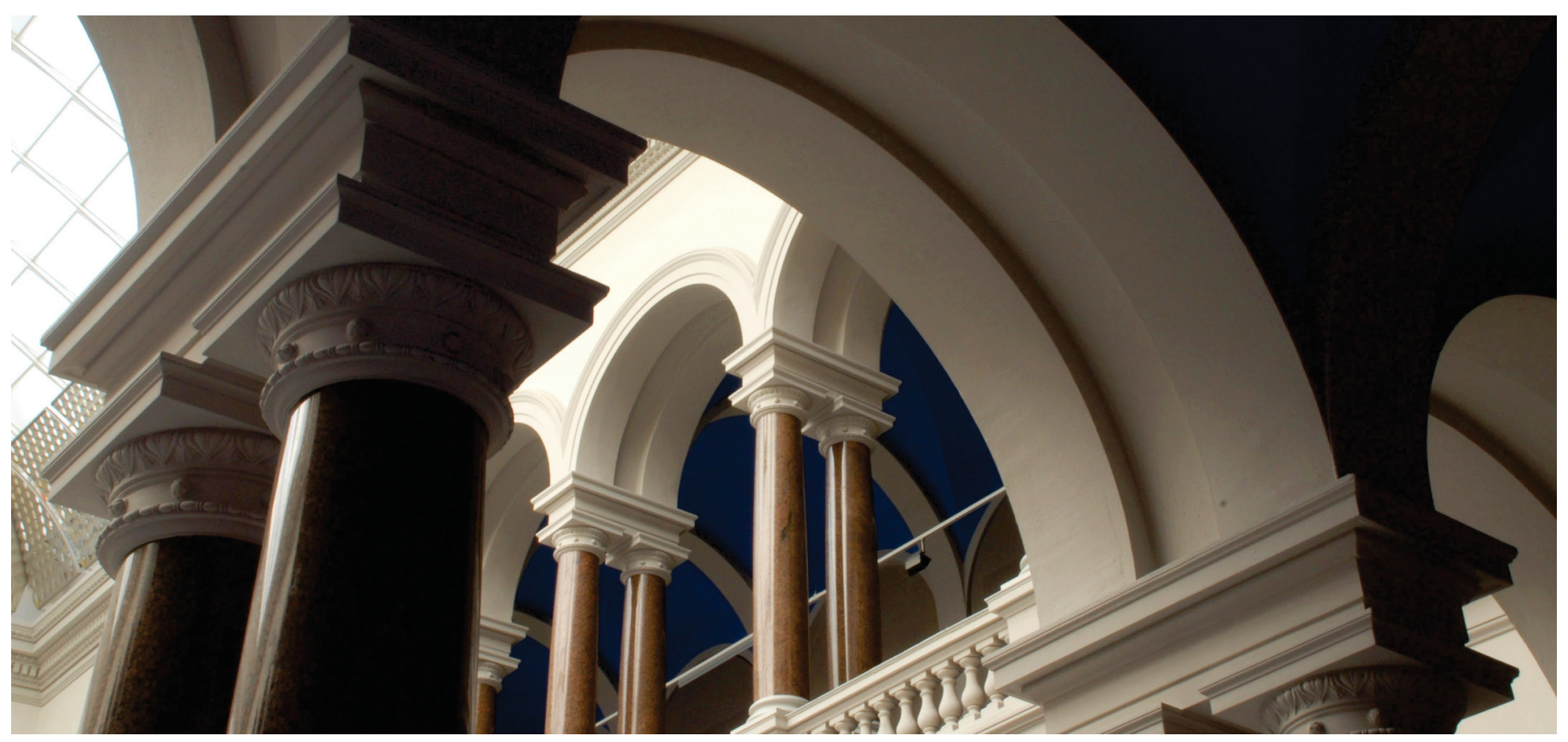

This is a post-peer-review, pre-copyedit version of an article published in Granular Matter. The final authenticated version is available online at: http://dx.doi.org/10.1007/s10035-017-0710-0

Elskamp, F., Kruggel-Emden, H., Hennig, M., \& Teipel, U. (2017). A strategy to determine DEM parameters for spherical and non-spherical particles. Granular Matter, 19(3).

https://doi.org/10.1007/s10035-017-0710-0 


\section{A strategy to determine DEM parameters for spherical and}

2 non-spherical particles

3 Frederik Elskamp ${ }^{1 \star}$, Harald Kruggel-Emden ${ }^{1}$, Manuel Hennig $^{2}$, Ulrich Teipel ${ }^{2}$

$4{ }^{1}$ Mechanical Process Engineering and Solids Processing, Technische Universität Berlin, Ernst-

5 Reuter-Platz 1, D-10623 Berlin, Germany

6 '2Department of Particle Technology, Technical University Nürnberg, Wassertorstrasse 10, D90489 Nuremberg, Germany

*Corresponding author. Tel.: +49-234-32-23492; Fax: +49-234-32-14227

9 E-mail address: frederik.elskamp@tu-berlin.de

\section{Abstract}

In Discrete Element Method (DEM) simulations the choice of appropriate contact parameters is significant to obtain reasonable results. Particularly, for the determination of DEM parameters for non-spherical particles a general straightforward procedure is not available. Therefore, in a first step of the investigation here, methods to obtain the friction and restitution coefficients experimentally for single particles (Polyoxymethylene (POM) spheres and quartz gravel) will be introduced. In the following, these predetermined DEM coefficients are used as initial values for the adjustment of bulk simulations to respective experiments. In the DEM simulations, the quartz gravel particles are represented by non-spherical particles approximated by clustered spheres. The best fit approximation of the non-spherical particles is performed automatically by a genetic algorithm. In order to optimize the sliding and rolling friction coefficients for DEM simulations, the static and dynamic angle of repose are determined from granular piles obtained by slump tests and rotating drum experiments, respectively. Additionally, a vibrating plate is used to obtain the dynamic bed height which is mainly influenced by the coefficient of restitution. The adjustment of the results of the bulk simulations to the experiments is conducted automatically by an optimization tool based on a genetic algorithm. The obtained contact parameters are later used to perform batch-screening DEM simulations and lead to accurate results. This underlines the applicability of the in parts automated strategy to obtain DEM parameters for particulate processes like screening.

Keywords: Discrete element method (DEM); Parameter determination; Automatic adjustment of DEM parameters; Arbitrary shaped particles; Screening

\section{Introduction}

The process step screening is important to separate bulk material in a wide range of industrial applications, where particles of non-spherical shape are classified according to desired size 
class specifications $[1,2]$. In the interest of studying screening and its sub-processes in detail without performing extensive experimental tests the Discrete Element Method (DEM) dating back to Cundall and Strack [3] has been proved as a suitable tool [4-8]. To apply the DEM for complex processes like screening a proper calibration of DEM parameters and particle shape approximation has to be carried out. Several methods to calibrate DEM parameters have been proposed but particularly, for non-spherical particles general straightforward procedures with a high degree of automation are hardly available.

The first investigations published, addressing DEM parameter calibration, mostly concentrate on spherical particles without automated procedures. In one of them [9] Li et al. measured the coefficient of sliding friction in simple drag tests applying spheres and confirmed the DEM parameters by comparing quasi-two-dimensional hopper discharge and conical pile experiments with simulations. Based on this, Gryma and Wypych [10] as well as Chen et al. [11] applied particle clusters consisting of two spheres to measure the static angle of repose in a slump test to confirm and adjust the DEM parameters which had been determined in single particle tests before. Coetzee and Els [12] also applied clusters of two spheres and determined the DEM parameters in shear and compression tests before measuring the static angle of repose. The obtained values were later applied successfully to hopper discharge and bucket filling processes. Natsui et al. [13] determined the contact friction and used rolling friction to consider the irregular shape of coke in simulations where the angle of repose was measured. They stated that the choice of the Young's modulus is important to accelerate the calculation time in DEM simulations as long as the results are not influenced. Alonso-Marroquín et al. [14] determined the DEM parameters stiffness, the friction and restitution coefficients for polygonal wood particles with several single particle experiments, including sliding, triaxial and pendulum collision tests, respectively. They applied the obtained parameters to hopper discharge simulations and compared the results with respective experiments. Similar single particle tests are carried out by Barrios et al. [15] applying particle clusters consisting of four different sized spheres to approximate iron ore pellets. The validation of the DEM parameters by slump and tumbling tests clarified the significance of particle shape and the need of adjusting the DEM parameters after simple single particle tests. A review for DEM parameters and contact models for granular material has been done by Horabik and Molenda [16] who highlighted the importance of material and interaction properties for obtaining reliable information out of DEM simulations. Recently, Coetzee [17] investigated the influence of particle shape approximation with particle clusters created manually and by an automated optimization process with the multi-sphere method of up to eight spheres as main part of a DEM parameter calibration. Additionally, Dobrohotoff et al. [18] used spheropolygons to describe two-dimensional complex-shaped objects like pebbles, gravel and crushed shells where only a few iteration 
steps were needed to get good results. The significant optimization of the shape was confirmed by comparing anchor pull-out and hopper discharge simulations with respective experiments. Höhner et al. $[19,20]$ investigated the influence of the multi-sphere and the polyhedral method on the mechanical behavior of particles during hopper discharge. Only minor differences were obtained between both methods for complex shaped particles. However, the computational time increased significantly if the polyhedral method was used for real particles like quartz gravel. Accordingly, Li et al. [21] approximated real particles with the multi-sphere method automatically by a greedy heuristic algorithm obtaining very accurate results. In order to determine adequate DEM parameters automatically, Benvenuti et al. [22] developed a neural network which can be trained by dedicated DEM simulations to predict granular bulk behavior for a large number of DEM parameter sets.

Based on these previous investigations, the strategy to determine DEM parameters in this investigation is as follows. First, DEM parameters and physical properties of single particles are determined. Additionally, non-spherical particles are clustered with the multi-sphere method automatically. Afterwards, the physical properties and the DEM parameters are applied to bulk simulations where the DEM parameters are adjusted automatically with an optimization tool to fit the results of corresponding experiments. To confirm the adjustment procedure, initial and adjusted parameter values are compared against each other. The adjusted DEM parameters are then applied to screening processes as example for a more complex particulate process.

\section{Numerical method}

The DEM can be applied to systems with spherical and non-spherical shaped particles [23,24], by tracking the translational and rotational motion of each particle. For this purpose the Newton's and Euler's equations are integrated

$$
\begin{aligned}
& m_{i} \frac{d^{2} \vec{x}_{i}}{d t^{2}}=\vec{F}_{i}+m_{i} \vec{g}, \\
& \hat{I}_{i} \frac{d \vec{\omega}_{i}}{d t}+\vec{\omega}_{i} \times\left(\hat{I}_{i} \vec{\omega}_{i}\right)=\Lambda_{i}^{-1} \vec{M}_{i},
\end{aligned}
$$

with particle mass $m_{i}$, particle acceleration $d^{2} \vec{x}_{i} / d t^{2}$, contact force $\vec{F}_{i}$, gravitational force $m_{i} \vec{g}$, angular acceleration $d \vec{\omega}_{i} / d t$, angular velocity $\vec{\omega}_{i}$, external moments resulting out of contact forces $\vec{M}_{i}$, the inertia tensor along the principal axis $\hat{I}_{i}$ and the rotation matrix converting a vector from the inertial into the body fixed frame $\Lambda_{i}^{-1}$. Explicit integration schemes (comp. e.g. [25]) are used to solve both equations (eq. (1) and eq. (2)). 
To model complex shaped particles in the DEM the multi-sphere method is used. Thereby, the desired complex particle shape is represented by clustered spheres of arbitrary size [26] and

101 similar contact force laws as used for spherical particles are applied [27].

$$
\gamma^{n}=-\left(2 \ln \left(e^{n}\right) m_{e f f}\right) / t^{n}
$$

Fig. 1: A collision of two multi-sphere particles.

In Fig. 1 a sketch of two complex shaped particles $i$ and $j$ is shown, where the spheres $I$ and $k$ collide. For further details on the contact scheme involving clustered spheres the works by Kruggel-Emden and Kačianauskas and Kruggel-Emden et al. [28,29] are recommended.

The normal component of the contact forces is obtained from a linear spring damper model which is exemplarily given for the contacting spheres $k$ and $I$ of particle $i$ and $j$ as

$$
\vec{F}_{i k j l}^{n}=k^{n} \delta_{i k j l} \vec{n}_{i k j l}+\gamma^{n} \vec{v}_{i k j l}^{n},
$$

where $k^{n}$ is the spring stiffness, $\delta_{i k j l}$ the virtual overlap, $\vec{n}_{i k j l}$ a normal vector, $\gamma^{n}$ a damping coefficient and $\vec{v}_{i k j l}^{n}$ the normal velocity at the contact point [30]. The damping coefficient $\gamma^{n}$ is calculated as

112 with the experimentally determined coefficient of restitution $e^{n}$ (comp. section 3.5), the duration 113 of a collision

$$
t^{n}=\pi /\left(\sqrt{\left(k_{n} / m_{e f f}-\left(\gamma_{n} /\left(2 m_{e f f}\right)\right)^{2}\right)}\right)
$$

114 and the effective mass $m_{\text {eff }}=m_{i} m_{j} /\left(m_{i}+m_{j}\right)$.

115 The tangential forces are calculated by applying a linear spring limited by the Coulomb 116 condition

$$
\vec{F}_{i k j l}^{t}=\min \left(k^{t}\left|\vec{\xi}_{i k j l}\right|, \mu_{C}\left|\vec{F}_{i k j l}^{n}\right|\right) \vec{t}_{i k j l},
$$


117 where $k^{t}$ is the tangential stiffness of a linear spring, $\mu_{C}$ is the friction coefficient, $\vec{\xi}_{i k j l}$ is the 118 relative tangential displacement and $\vec{t}_{i k j l}$ is the tangential unit vector [31]. The tangential spring 119 stiffness $k^{t}$ is obtained from

$$
k^{t}=\kappa m_{e f f}\left(\pi / t^{n}\right)^{2}
$$

120 where $\kappa$ is given through the mechanical properties as

$$
\kappa=\left(\left(1-v_{i}\right) / G_{i}+\left(1-v_{j}\right) / G_{j}\right) /\left(\left(1-0.5 v_{i}\right) / G_{i}+\left(1-0.5 v_{j}\right) / G_{j}\right)
$$

121 where $v$ is the Poisson's ratio and $G=E /(2+2 v)$ with Young's modulus $E$ is the shear 122 modulus of the two interacting materials $i$ and $j[32]$.

\section{Determination of DEM parameters and physical properties of single particles}

In this section, the material and physical properties, the particle shapes, the sliding and rolling friction coefficients and the coefficient of restitution are obtained by single particle characterization. In case of the coefficients of friction and restitution the calibration at the particle level is done to get a first approximation. These parameters can be used as initial values for the parameter adjustment so that the calibration converges quickly; additionally they are used as comparison for the values reached after the adjustment. If these values are already accurate enough, the adjustment is very fast or is not necessary at all.

3.1 Determination of material and physical properties

In a first step, material and physical properties like the size distribution, mass, volume and density of the particles are determined and listed in Table 1.

134 Table 1: Mechanical particle and wall properties.

\begin{tabular}{c|cc|cc}
\hline & \multicolumn{2}{|c}{ Particle } & \multicolumn{2}{c}{ Wall } \\
\hline Mechanical particle property & POM & Gravel & Metal & Acryl \\
\hline Diameter $d[\mathrm{~mm}]$ & $5 / 7 / 10 \pm 0.1$ & $3.15-5.60$ & - & - \\
Mass $m[\mathrm{~g}]$ & $0.0935 / 0.2459 / 0.7210 \pm 0.02$ & $0.0316-0.4440$ & - & - \\
Density $\rho\left[\mathrm{kg} / \mathrm{m}^{3}\right]$ & $1.43 \mathrm{E}+03 / 1.37 \mathrm{E}+03 / 1.38 \mathrm{E}+03 \pm 1.50 \mathrm{E}+03$ & $2.76 \mathrm{E}+03$ & $7.85 \mathrm{E}+03$ & $1.20 \mathrm{E}+03$ \\
Young's modulus $E\left[\mathrm{~N} / \mathrm{m}^{2}\right]$ & $2.84 \mathrm{E}+09$ & $6.00 \mathrm{E}+10$ & $2.08 \mathrm{E}+11$ & $2.20 \mathrm{E}+09$ \\
Poisson's ratio $v[-]$ & 0.35 & 0.25 & 0.30 & 0.37 \\
Stiffness $k_{P P}^{n} / k_{P W}^{n}[\mathrm{~N} / \mathrm{m}]$ & $1.00 \mathrm{E}+05$ & $1.00 \mathrm{E}+05$ & - & - \\
\hline
\end{tabular}

136 Polyoxymethylene (POM) spheres are applied in three different discrete size classes which 137 are equally distributed in each investigated case. For the quartz gravel the cumulative particle 138 size distribution as outlined in Fig. 2 which is obtained by an image analysis of a CAMSIZER ${ }^{\circledR}$ is used. Note that the dots represent one size class in the DEM simulations. 


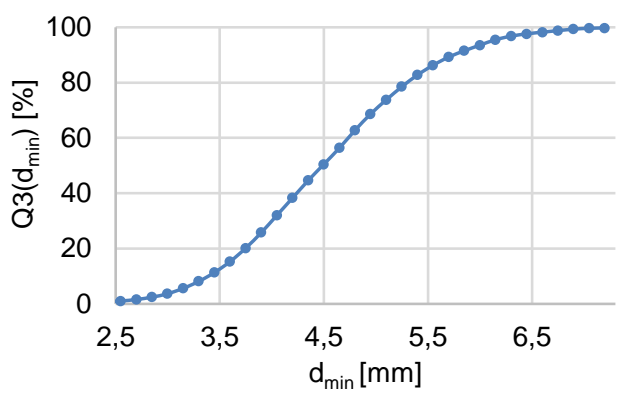

Fig. 2: Cumulative particle size distribution of quartz gravel.

142 The mass $m_{i}$ of each POM particle size class is measured as average of 20 POM spheres and 143 the density $\rho_{i}$ is determined with $\rho_{i}=m /\left(1 /\left(6 \pi d^{3}\right)\right)$. The average volume $V_{i}$ of the gravel is 144 obtained by water displacement of around 500 particles which are weighed before to obtain an average density of $\rho=2755 \mathrm{~kg} / \mathrm{m}^{3}$. The normal spring stiffness is set to $k^{n}=1.00 \mathrm{E}+05 \mathrm{~N} / \mathrm{m}$ for both materials after carrying out test simulations of granular piles (comp. section 4.2) applying values of $k^{n}=1.00 \mathrm{E}+03 \mathrm{~N} / \mathrm{m}$ up to $k^{n}=1.00 \mathrm{E}+06 \mathrm{~N} / \mathrm{m}$ and measuring no changes for values larger than $1.00 \mathrm{E}+05 \mathrm{~N} / \mathrm{m}$. Additionally, the particle overlap is consistently below $0.5 \%$ of the particle diameter according to Cleary [33].

\subsection{Shape approximation}

151 As stated before, the POM particles are assumed to be ideal spheres of $5 \mathrm{~mm}, 7 \mathrm{~mm}$ and $15210 \mathrm{~mm}$ which are applied in the DEM simulations accordingly. The gravel consists of non153 spherical particles with a sphericity between 0.35 and 0.87 (average: 0.75 ) and an aspect ratio between 1.06 and 2.49 (average: 1.49) which is both obtained by an image analysis. To represent such particles, various methods can be applied $[17,18,21]$. For this approximation a genetic algorithm which is part of Matlab is used. This algorithm is very flexible and allows adjusting many features such as population size, generation, mutation and crossover functions as well as initial values (comp. [37]). It was already applied successfully in the work by Kruggel-

159 Emden et al. [34] for the adjustment of coefficients as part of multi-parameter models describing reaction kinetics in the context of chemical looping where details on the algorithm and possible settings can be found. Instead of modelling the gravel with a polyhedral shape, the particles are approximated by clustered spheres like in Coetzee et al. [17] to save computational time in the DEM simulation, which is important for an efficient adjustment 164 algorithm.

a) side $A$ side B side C

b) side $A$ side $B$ side C c) 3D view
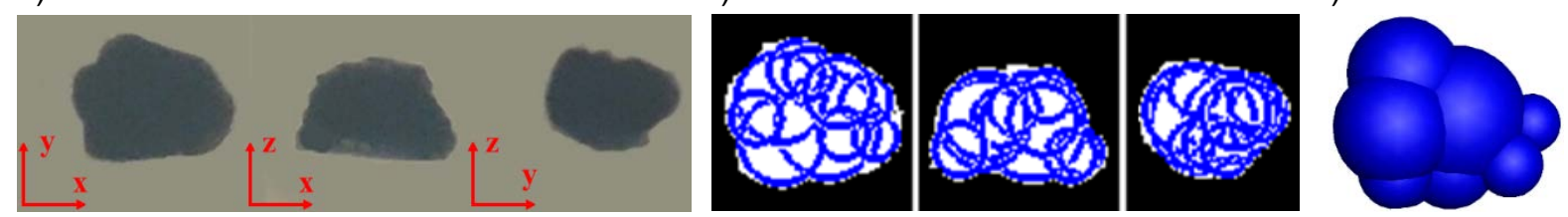

Fig. 3: (a) Cross sectional areas and (b) areas filled with circles to (c) approximate the shape of gravel particles (example). 
Therefore, in a first step, a sample of gravel particles is filled into cast resin cubes and images are taken from three sides giving the three cross sectional areas (Fig. 3a). With the help of an optimization tool, arbitrary sized circles, which represent spheres, are placed one after the other into these three areas to obtain the best fit for all areas while receiving penalty points for not included pixels in or for overlaps over a projected zone (Fig. 3b). The number of applied spheres determines the accuracy of the approximation but in contrary influences the simulation time. One method to terminate the optimization is to specify the amount of clustered spheres before the optimization, whereas another method is to automatically stop the tool if no further improvement is detected when applying more spheres (decrease in deviation is lower than defined). For the placing of each single sphere, the maximum number of iterations or a specified change in deviations to the previous iteration can be defined. The whole algorithm is presented in the flow chart in Fig. 4.

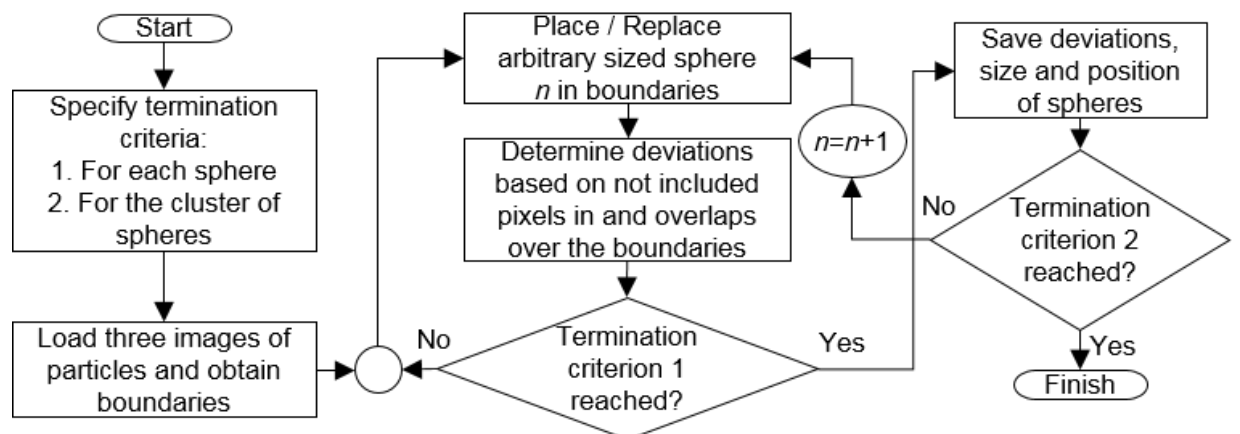

Fig. 4: Algorithm to best fit the representation of gravel particles with clustered spheres on the basis of cross-sectional areas.

179 One of the created gravel particles which is applied in the following simulations is shown in

180 Fig. 3c. It consists of 10 spheres which is sufficient enough to represent the gravel particles

181 (no further improvement by applying more spheres) and is still computationally efficient. It is 182 accomplished in less than 10 minutes requiring less than 100 generations where a population 183 consisting of 200 individuals was used. For the simulations, several different shapes for each 184 size class can be applied. A particle can be classified in one particle size class by measuring its volume equivalent sphere diameter if the particle size distribution is based on this equivalent diameter. Due to applying the particles for a screening process in the following, the approximated particle is classified based on the minor axis diameter in this investigation (comp.

188 Fig. 2). The other particle size classes are created by scaling the approximated cluster with 189 respective scaling factors obtained through the ratio of the equivalent diameters of two size 190 classes.

\subsection{Coefficient of sliding friction}

192 To determine the sliding friction of the particles, samples of POM spheres of each size class and gravel particles of each fraction are glued under a wooden plate and the force which is necessary to drag these particles over a plate consisting of the material of one contact partner 
is measured (comp. Fig. 5). Thereby, the evenness of the particle layer influences the quality 196 of the measurement. To stabilize the wooden plate a weight is placed on it. The value of the 197 weight is changed to test its influence resulting in deviations of below $1 \%$.

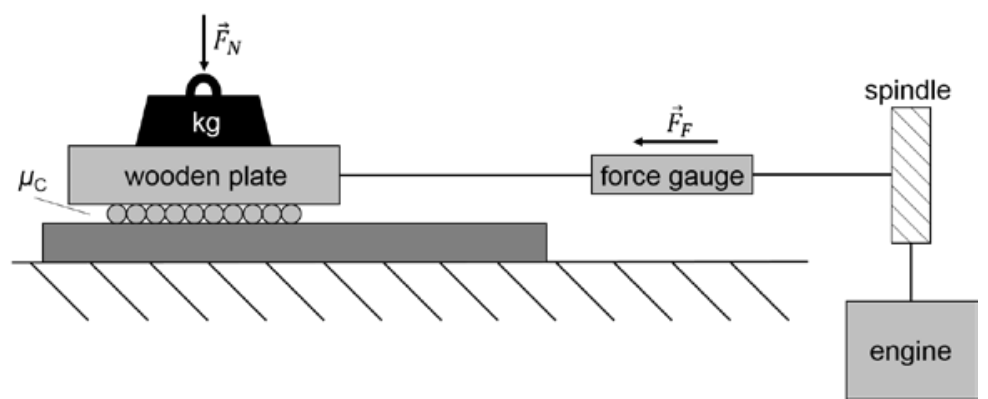

198

199

200

201

202

203

204

205

206

207

208

209

210

211

212

213

214

215

216

Fig. 5: Determination of sliding friction.

In this investigation, the classical friction theory is applied, which states that $\mu_{c}=\left|\vec{F}_{F}\right| /\left|\vec{F}_{N}\right|$, with the frictional force $\vec{F}_{F}$ and the normal force $\vec{F}_{N}$. This is suitable for a point-point contact when ignoring the effect of the contact area [9]. Due to having a very small contact area between a particle and a plane wall like between two particles, a plate of the particle material (POM spheres with a POM plate and quartz gravel with a gravel plate) is used as approximation for the particle-particle friction.

Results for the sliding friction coefficient between particles and the same material for the particle-particle contacts and between particles and the wall materials (acryl, metal) for the particle-wall contacts each averaged over 10 experiments are listed in Table 2. For two different sized POM spheres the average of the respective values is applied.

Table 2: Coefficient of sliding friction.

\begin{tabular}{c|cccc}
\hline Contact partner & POM $5 \mathrm{~mm}$ & POM 7 mm & POM $10 \mathrm{~mm}$ & Gravel \\
& $\mu_{c}$ & $\mu_{c}$ & $\mu_{c}$ & $\mu_{c}$ \\
\hline Acryl (side walls) & $0.16 \pm 0.01$ & $0.19 \pm 0.01$ & $0.21 \pm 0.01$ & $0.19 \pm 0.01$ \\
Metal (screen wire) & $0.17 \pm 0.01$ & $0.16 \pm 0.01$ & $0.17 \pm 0.02$ & $0.31 \pm 0.02$ \\
POM / quartz gravel (particles) & $0.23 \pm 0.01$ & $0.30 \pm 0.02$ & $0.28 \pm 0.02$ & $0.57 \pm 0.03$ \\
\hline
\end{tabular}

\subsection{Coefficient of rolling friction}

For simulating spherical particles in the DEM (here for POM spheres) it is necessary to use a model for rolling friction to oppose the rolling motion of the spheres with a decelerating moment $\vec{M}_{\text {roll }}$. Here, the model by Zhou et al. [35] is used which can be simplified for free rolling spherical particles where the normal force $\vec{F}_{N}$ is equal to the weight force. Therefore, the coefficient of rolling friction can be calculated as

$$
\mu_{\text {roll }}=\left|\vec{M}_{\text {roll }}\right| /\left|\vec{F}_{N}\right| .
$$


217 To determine the coefficient of rolling friction experimentally, the rolling motion of a sphere on

218 a plane wall is recorded [36]. The moment $\vec{M}_{\text {roll }}$ is then obtained as

$$
\left|\vec{M}_{\text {roll }}\right|=\left(1 / 2 m\left(v_{1}^{2}-v_{2}^{2}\right)+1 / 2 \theta\left(\omega_{1}^{2}-\omega_{2}^{2}\right)\right) d /(2 s)
$$

219 with the velocities of the sphere at the beginning $\left(v_{1}\right)$ and at the end $\left(v_{2}\right)$ of the surface, the 220 sphere's mass moment of inertia $\theta$, its angular velocity at both time steps $\omega_{1}$ and $\omega_{2}$, the 221 distance travelled by the sphere $s$ and its half diameter $d / 2$. The velocities of the sphere $v_{1}$ and $222 v_{2}$ as well as the distance travelled are measured by image analysis of records taken from 223 above with a high speed camera. It can be assumed that the spheres do not slip or bounce on 224 the applied plane surface and for the low velocities. Therefore, the angular velocity $\omega$ can be obtained through the translational velocity $v$.

226 Results for the coefficient of rolling friction between POM spheres and a POM plate for the 227 particle-particle contacts and between particles and the wall materials (acryl, metal) for the 228 particle-wall contacts each averaged over 10 experiments are listed in Table 3. For two 229 different sized POM spheres the average of the respective values is applied. The large 230 standard deviations indicate the need of a further adjustment of these initial values.

231 Table 3: Coefficient of rolling friction.

\begin{tabular}{c|ccc}
\hline Contact partner & POM $5 \mathrm{~mm}$ & POM $7 \mathrm{~mm}$ & POM $10 \mathrm{~mm}$ \\
& $\mu_{\text {roll }}\left[10^{-5} \mathrm{~m}\right]$ & $\mu_{\text {roll }}\left[10^{-5} \mathrm{~m}\right]$ & $\mu_{\text {roll }}\left[10^{-5} \mathrm{~m}\right]$ \\
\hline Acryl (side walls) & $5.44 \pm 1.1$ & $7.76 \pm 2.7$ & $6.41 \pm 3.3$ \\
Metal (screen wire) & $7.22 \pm 2.2$ & $8.13 \pm 3.6$ & $7.07 \pm 3.7$ \\
POM (particles) & $5.94 \pm 1.4$ & $8.63 \pm 1.6$ & $7.76 \pm 3.1$ \\
\hline
\end{tabular}

232

\subsection{Coefficient of restitution}

234 In order to determine the coefficient of restitution, a particle which is on the end of a pendulum 235 is dropped so that it bounces against a wall (comp. Fig. 6a) or another particle which has a 236 velocity of $\mathrm{v}_{2}=0$ before the collision according to Alonso-Marroquín et al. [14] (comp. Fig. 6b). 237 For the particle wall collisions this experimental setup was chosen instead of a drop test to 238 have the same external effects in both experiments. Note that the procedure in Fig. $6 \mathrm{~b}$ is only 239 applied for POM spheres, whereas for quartz gravel particles the setup in Fig. 6a is used with 240 a wall of the same material due to their arbitrary shapes, resulting in rotations or skewed 241 rebounds. Furthermore, it should be mentioned, that these experiments are more difficult if 242 smaller particles with a low mass compared to the mass of the thread are applied. 
a

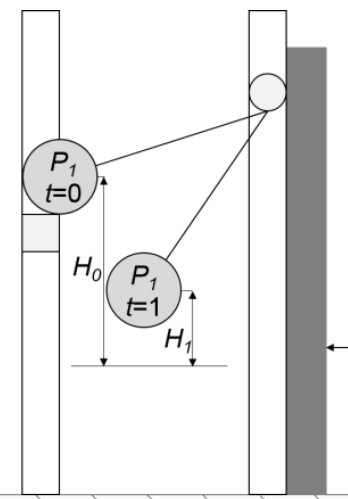

Wall out of material

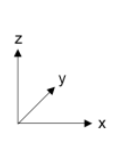

b of contact parameter

Fig. 6: Determination of the restitution coefficient for (a) particle-wall and (b) particle-particle contacts according to AlonsoMarroquín et al. [14].

245 For the particle-wall contact, the velocities before $\left(u_{1}\right)$ and after the rebound $\left(v_{1}\right)$ are measured. Alternatively, the heights before the particle drop $\left(H_{0}\right)$ and at the highest point after the rebound $\left(H_{1}\right)$ could be measured. Thus, the particle-wall restitution coefficient is obtained by

$$
e_{P W}^{n}=-v_{1} / u_{1}=\sqrt{H_{1} / H_{0}} .
$$

The restitution coefficient for a particle-particle contact is determined by

$$
e_{P P}^{n}=-\left(v_{1}-v_{2}\right) / u_{1}=\left(\sqrt{H_{2}}-\sqrt{H_{1}}\right) / \sqrt{H_{0}}
$$

where $u_{1}$ and $v_{1}$ are the velocities and $H_{0}$ and $H_{1}$ the heights of particle $P_{1}$ before and after the collision, respectively. Furthermore, $v_{2}$ is the velocity and $H_{2}$ the height of particle $P_{2}$ after the collision with particle $P_{1}$.

Results for the coefficient of restitution between particles and the same material (each size of POM sphere with itself and the other sizes and quartz gravel with a gravel plate) for the particleparticle contact and between particles and the wall materials (acryl, metal) for the particle-wall contacts are listed in Table 4.

Table 4: Coefficient of restitution.

\begin{tabular}{cc|ccc|cc|c}
\hline Contact partner & & POM $5 \mathrm{~mm}$ & POM $7 \mathrm{~mm}$ & POM $10 \mathrm{~mm}$ & Contact partner & Gravel \\
\hline Acryl (side walls) & $e_{P W}^{n}$ & $0.88 \pm 0.01$ & $0.76 \pm 0.01$ & $0.81 \pm 0.05$ & Acryl (side walls) & $e_{P W}^{n}$ & $0.81 \pm 0.01$ \\
Metal (screen wire) & $e_{P W}^{n}$ & $0.80 \pm 0.01$ & $0.83 \pm 0.01$ & $0.74 \pm 0.04$ & Metal (screen wire) & $e_{P W}^{n}$ & $0.48 \pm 0.02$ \\
POM (5 mm particle) & $e_{P P}^{n}$ & $0.84 \pm 0.02$ & $0.81 \pm 0.04$ & $0.85 \pm 0.03$ & $\begin{array}{c}\text { Quartz gravel plate } \\
\text { (gravel particle) }\end{array}$ & $e_{P P}^{n}$ & $0.77 \pm 0.01$ \\
POM (7 mm particle) & $e_{P P}^{n}$ & $0.81 \pm 0.04$ & $0.82 \pm 0.02$ & $0.86 \pm 0.03$ & & \\
POM (10 mm particle) & $e_{P P}^{n}$ & $0.85 \pm 0.03$ & $0.86 \pm 0.03$ & $0.87 \pm 0.03$ & & \\
\hline
\end{tabular}

\section{Adjustment of DEM parameters of particles as bulk material}

After the calibration at the particle level in section 3 the parameters have to be adjusted based on the bulk behavior due to two reasons. Firstly, it could be possible, that due to particle size 
or shape it is not possible to perform an accurate calibration at the single particle level where assumptions have to be made. A second aspect is that parameter sets obtained at the single particle level and at the bulk level differ from each other. This is a result of inaccuracies related to the single particle measurements for which it is compensated for in the bulk calibration or of the models (e.g. insufficient approximation of shape or of contact models). In a first step before the adjustment, three different bulk experiments with several settings are conducted. They are later compared with respective simulations to test the DEM parameters obtained by the single particle experiments before adjusting the DEM parameters to minimize the differences between the results of simulations and experiments. In the bulk experiments the static and dynamic angles of repose which are mainly influenced by the friction coefficients between particles (static) and particles and walls (dynamic) are measured. Furthermore, the bed height on a vibrating plate is determined, where the restitution coefficient is the crucial parameter. Note that in all investigations with POM spheres the same mass for each fraction and for gravel the particle size distribution from Fig. $\mathbf{2}$ is applied. In all investigations the appliances are filled according to a defined filling degree or level. Due to different bulk densities in case of POM spheres, the resulting average particle mass varies slightly dependent on the applied particle size classes.

\subsection{Adjustment of DEM parameters by a genetic algorithm}

For obtaining the best fit between simulations and experiments for all investigated bulk experiments, an optimization tool based on a genetic algorithm [37] like the one used for the shape approximation in section 3.2 is used. The whole adjustment procedure for one particle distribution and an arbitrary number of bulk calibration processes is outlined in Fig. 7. Therein, the termination criterion can be a defined number of generations or a specified change in deviations between two generations. The algorithm is fed with the results shown in Fig. 9, Table 7 and Table 9 and the initial DEM parameters listed in Table 1, Table 2 and Table 4. The DEM parameters are changed in defined physical boundaries, supporting a quick convergence of the optimization. As DEM simulations are dependent on input parameters in a complex way the generations required for convergence are varying. It dependents on the quality of the initial values, their difference to the values after optimization and if the initial values lead to consistent results. At the most 100 generations were enough to get good results which were not further improved in consecutive generations; often 50 generations were sufficient. Due to calculating up to 100 generations for each adjustment, one main criterion is the time needed for one simulation. Thus, the dimensions of the bulk tests are chosen as small as possible while still obtaining suitable results. 


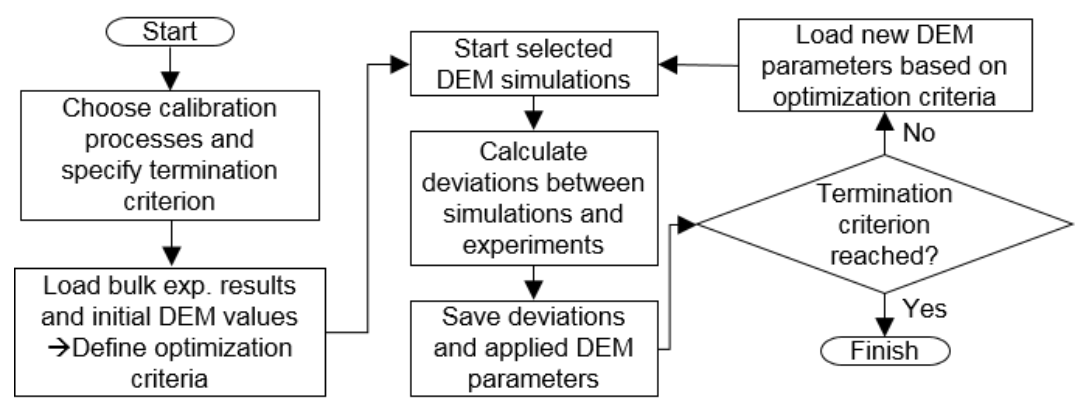

Fig. 7: Algorithm to adjust the DEM parameters for the best fit between simulations and experiments.

296 In case of the gravel particles the procedure is performed one time for all size classes due to 297 the simplification of using the same friction and restitution coefficients for each particle. In case 298 of the POM spheres with three discrete size classes, one possible method would be to directly 299 fit the DEM parameters for all size classes. However, in this investigation, all monodisperse cases are fitted before the bidisperse cases to reduce the amount of adjustable parameters in one adjustment procedure and thereby, to enhance the quality of the optimization. The obtained parameters are then applied for the case with three different particle size classes.

\subsection{Static angle of repose}

Static angle of repose measurements were conducted in a slump test by releasing POM spheres and gravel particles contained in a hollow acrylic cylinder onto an acrylic and a steel surface (Fig. 8a). To prevent excessive spreading of spheres, an acrylic ring as boundary is used for both materials. The static angle of repose $\alpha_{s t a}$ is measured after reaching a steady state. The experimental and simulative properties (Fig. 8b,c) are listed in Table 5.

Table 5: Experimental and simulative properties for measuring the static angle of repose.

\begin{tabular}{c|cc}
\hline Properties & POM & Gravel \\
\hline Bed height $[\mathrm{m}]$ & 0.065 & 0.040 \\
Cylinder velocity $[\mathrm{m} / \mathrm{s}]$ & 0.011 & 0.011 \\
Cylinder diameter $[\mathrm{m}]$ & 0.070 & 0.035 \\
Ring diameter $[\mathrm{m}]$ & 0.125 & 0.072 \\
Particle mass $[\mathrm{kg}]$ & $0.195-0.221$ & 0.060 \\
\hline
\end{tabular}

311 For the simulations, DEM parameters obtained in section 3 are applied in the first step of the 312 adjustment. Therein, the angle of repose is mainly influenced by the rolling (in case of spheres) 313 and sliding friction coefficient between particles. This simulation is time-determining for the 314 whole adjustment procedure, due to needing the longest time for reaching the steady state. 315 Therefore, it should be calculated with a larger number of processors $n_{\text {proc }}$ as used for the other 316 simulations ( $n_{\text {prod }} / 2$ for the tumbling and approx. $n_{\text {prod }} / 4$ for the vibrating plate simulation). Note, 317 that the DEM code used is parallelized using domain decomposition. 
a $\quad v=0.011 \mathrm{~m} / \mathrm{s} \quad \mathrm{b}$
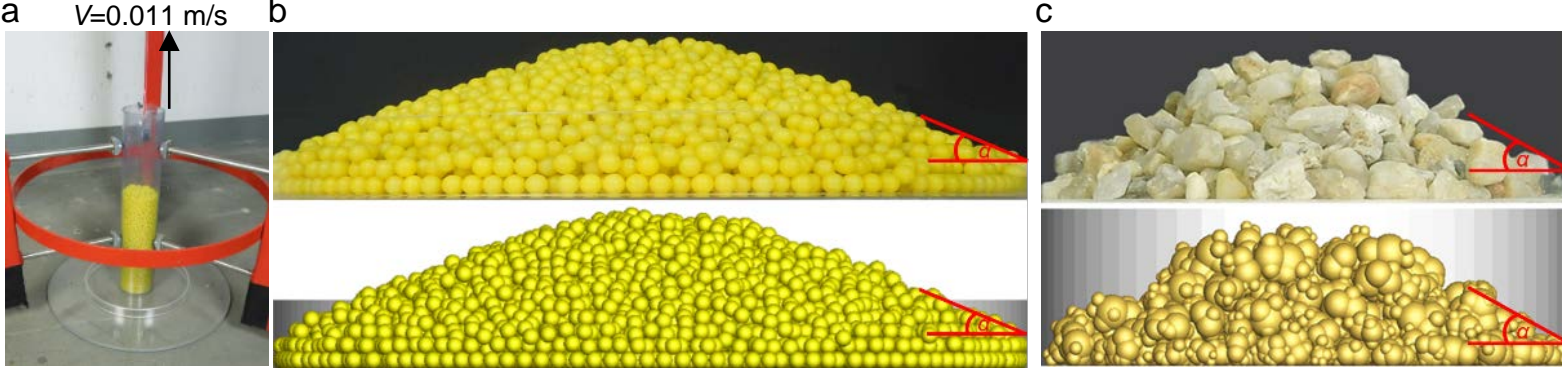

Fig. 8: (a) Experimental set-up to measure the static angle of repose and (b) resulting piles of $5 \mathrm{~mm}$ POM spheres as well as (c) piles of gravel in the experiments (top) and the simulations (bottom).

Most of the results for POM spheres and for gravel particles applying the initial DEM parameters reveal a good agreement between simulations and experiments with deviations below the standard deviations (comp. Fig. 9). In contrast, the simulations with $7 \mathrm{~mm}$ and $10 \mathrm{~mm}$ POM spheres (not shown in Fig. 9) and with 3 size classes applying an acryl surface form out too flat static angles of repose.

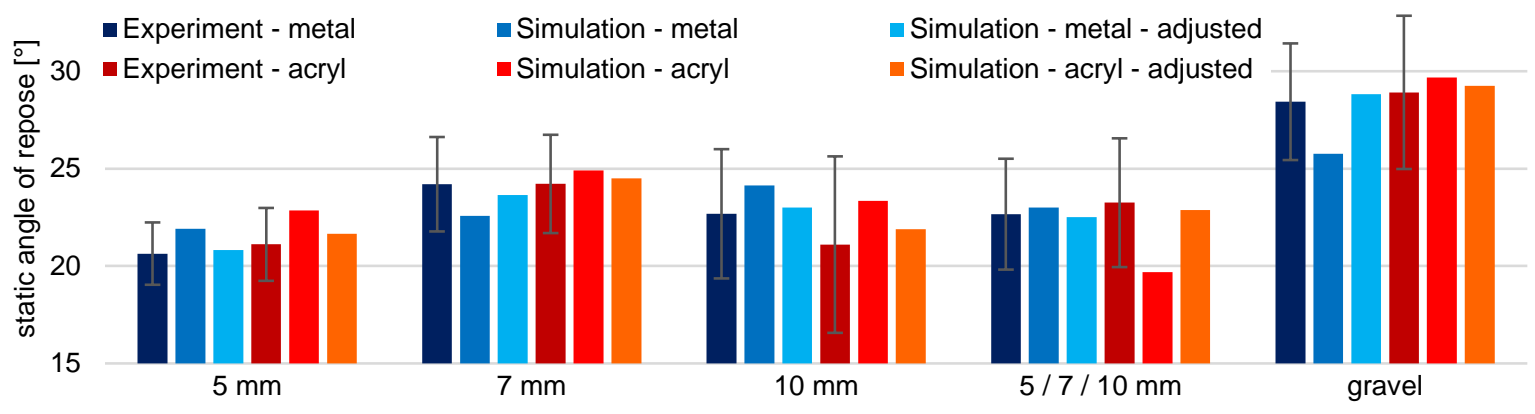

Fig. 9: Experimental results of measuring the static angle of repose $\alpha_{\text {sta }}$ and comparison to simulations applying DEM parameters determined in section 3 as well as after the adjustment.

327 By adjusting the DEM parameters (comp. Table 10), the deviations are reduced for all cases 328 but particularly for the polydisperse simulations where $7 \mathrm{~mm}$ and $10 \mathrm{~mm}$ spheres are in contact 329 (averagely from $9.59 \%$ to $2.15 \%$ ).

\subsection{Dynamic angle of repose}

331 For determining the dynamic angle of repose $\alpha_{d y n}$, tumbling tests with a hollow acrylic and a metal cylinder (Fig. 10a) are conducted for POM spheres (Fig. 10b,c) and gravel particles (Fig. 10d,e) with three different velocities and $30 \%$ filling (comp. Table 6). The dynamic angle of repose was measured at 10 different points in time after a transient period of one second.

Table 6: Experimental and simulative properties for measuring the dynamic angle of repose.

\begin{tabular}{c|cc}
\hline Properties & POM & Gravel \\
\hline Cylinder velocity $[\mathrm{rpm}]$ & \multicolumn{2}{|c}{$10 / 15 / 20$} \\
Cylinder diameter $[\mathrm{m}]$ & \multicolumn{2}{|c}{0.036} \\
Cylinder depth $[\mathrm{m}]$ & \multicolumn{2}{|c}{0.250} \\
Filling degree $[\%]$ & 30 \\
Particle mass $[\mathrm{kg}]$ & $0.061-0.069$ & 0.130 \\
\hline
\end{tabular}


a

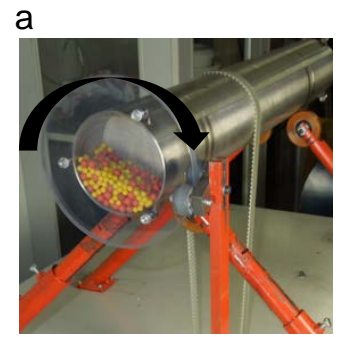

b c

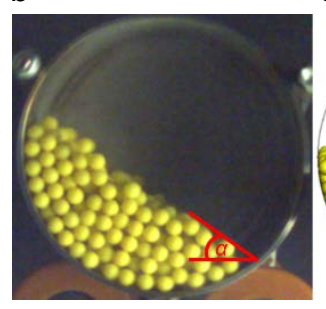

c

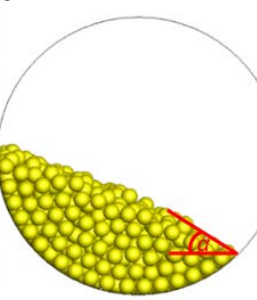

d

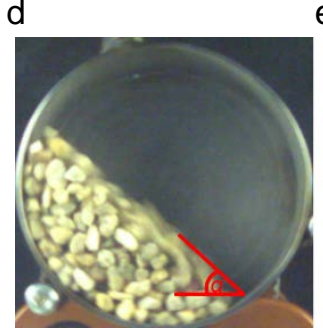

e

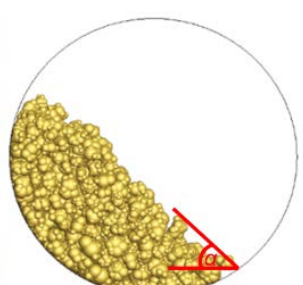

337

338

339

340

341

342

343

344

345

346

Fig. 10: (a) Experimental set-up to measure the dynamic angle of repose as well as resulting piles of (b,c) $5 \mathrm{~mm}$ POM spheres and $(d, e)$ gravel in $(b, d)$ the experiments and $(c, e)$ the simulations.

The results, which are mainly influenced by the friction coefficients between particles and walls are presented in Table 7. All investigations for POM spheres and for gravel with the initial DEM parameters for an acrylic cylinder obtained in section 3 reveal a much lower dynamic angle of repose in the simulations than in the experiments (average deviations of $26.39 \%$ ). The simulations with the metallic cylinder also reveal lower angles which are however closer to the experimental ones (average deviations of $18 \%$ ). The low initial friction coefficient between particles and acrylic walls leads to slip which is prevented to some extent by the larger coefficient in case of metallic walls.

Table 7: Experimental results of measuring the dynamic angle of repose $\alpha_{d y n}$ and comparison to simulations applying DEM parameters determined in section 3 as well as after the adjustment.

\begin{tabular}{|c|c|c|c|c|c|}
\hline \multirow{2}{*}{ Static angle of repose $\alpha_{d y n}\left[^{\circ}\right]$} & \multicolumn{4}{|c|}{ POM } & \multirow{2}{*}{$\begin{array}{c}\text { gravel } \\
3.15-5.6 \mathrm{~mm}\end{array}$} \\
\hline & $5 \mathrm{~mm}$ & $7 \mathrm{~mm}$ & $10 \mathrm{~mm}$ & $5 / 7 / 10 \mathrm{~mm}$ & \\
\hline Experiment - metal - $10 \mathrm{rpm}$ & $28.26 \pm 2.15$ & $31.73 \pm 4.06$ & $23.26 \pm 2.74$ & $32.03 \pm 3.76$ & $40.81 \pm 1.98$ \\
\hline Simulation - metal - $10 \mathrm{rpm}$ & 25.39 & 23.92 & 20.65 & 23.76 & 39.49 \\
\hline Simulation - metal - 10 rpm - adjusted & 29.79 & 32.92 & 23.15 & 30.64 & 41.49 \\
\hline Experiment - acryl - 10 rpm & $33.40 \pm 2.14$ & $38.81 \pm 1.34$ & $32.25 \pm 3.37$ & $33.91 \pm 2.61$ & $42.82 \pm 1.68$ \\
\hline Simulation - acryl - 10 rpm & 24.01 & 27.87 & 22.81 & 25.68 & 28.97 \\
\hline Simulation - acryl - 10 rpm - adjusted & 33.01 & 37.87 & 29.81 & 32.25 & 42.54 \\
\hline Experiment - metal - 15 rpm & $29.80 \pm 1.73$ & $32.16 \pm 4.32$ & $24.70 \pm 4.74$ & $31.31 \pm 3.51$ & $40.99 \pm 1.28$ \\
\hline Simulation - metal - $15 \mathrm{rpm}$ & 25.06 & 24.58 & 22.8 & 24.93 & 38.96 \\
\hline Simulation - metal - 15 rpm - adjusted & 29.49 & 33.91 & 25.49 & 31.17 & 41.16 \\
\hline Experiment - acryl - 15 rpm & $32.82 \pm 1.31$ & $35.75 \pm 3.88$ & $32.59 \pm 3.52$ & $34.85 \pm 2.53$ & $42.95 \pm 1.73$ \\
\hline Simulation - acryl - 15 rpm & 23.5 & 25.6 & 22.6 & 26.82 & 29.23 \\
\hline Simulation - acryl - 15 rpm - adjusted & 32.5 & 35.35 & 29.68 & 33.58 & 43.89 \\
\hline Experiment - metal - 20 rpm & $30.33 \pm 2.31$ & $29.87 \pm 2.89$ & $26.42 \pm 2.55$ & $32.58 \pm 2.38$ & $40.39 \pm 1.61$ \\
\hline Simulation - metal - $20 \mathrm{rpm}$ & 24.62 & 21.7 & 21.28 & 25.02 & 41.19 \\
\hline Simulation - metal - 20 rpm - adjusted & 29.06 & 30.7 & 24.78 & 32.39 & 41.59 \\
\hline Experiment - acryl - 20 rpm & $33.93 \pm 2.77$ & $35.29 \pm 2.56$ & $33.72 \pm 2.71$ & $34.29 \pm 2.33$ & $42.57 \pm 1.87$ \\
\hline Simulation - acryl - 20 rpm & 23.3 & 25.38 & 24.99 & 25.8 & 29.44 \\
\hline Simulation - acryl - 20 rpm - adjusted & 32.3 & 34.38 & 31.99 & 32.56 & 43.13 \\
\hline
\end{tabular}

After adjusting the DEM parameters (comp. Table 10) and particularly by increasing the friction coefficient between particles and walls, the deviations of the dynamic angle of repose are all minimized significantly and below the standard deviation (average deviations of $3.18 \%$ ). 


\subsection{Bed heights on a vibrating plate}

354

355

356

357

358

359

360

361

362

363

364

365

366

367

368

In order to obtain the dynamic bed height, tests on a vibrating metal plate with three different degrees of filling are conducted (comp. Fig. 11; here with one particle layer of $5 \mathrm{~mm}$ spheres or $5 \%$ filling) for POM spheres (Fig. 11b,c) and gravel particles (Fig. 11d,e). The average maximum bed height (referred to as "Top") and the average distance between the lowest particles and the bottom plate (referred to as "Bottom") at different points in time are measured using the properties listed in Table 8. Therein, the vibration parameters are obtained by an accelerometer which measures an amplitude of around $1.2 \pm 0.04 \mathrm{~mm}$ in z-direction and only minor amplitudes in the horizontal $(x-/ y$-stroke $<0.1 \mathrm{~mm})$ at a frequency of $54 \mathrm{~Hz}$. Note that the bed height is analyzed after one second when a continuous motion of the plate is ensured.

Table 8: Experimental and simulative properties for measuring the dynamic bed height.

\begin{tabular}{|c|c|c|}
\hline Properties & POM & Gravel \\
\hline Length, width and height [m] & \multicolumn{2}{|c|}{$0.045 \times 0.045 \times 0.100$} \\
\hline Amplitude [mm] & \multicolumn{2}{|c|}{$1.2 \pm 0.04$} \\
\hline Frequency $[\mathrm{Hz}]$ & \multicolumn{2}{|c|}{54} \\
\hline Stroke angle $\left[{ }^{\circ}\right]$ & \multicolumn{2}{|c|}{$\sim 90$} \\
\hline Filling degree [\%] & \multicolumn{2}{|c|}{1 Layer (5-10) / 20 / 50} \\
\hline Particle mass [kg] & $\begin{array}{c}0.007-0.013 / 0.029- \\
0.033 / 0.076-0.083\end{array}$ & $0.013 / 0.065 / 0.162$ \\
\hline
\end{tabular}

The experimental results presented in Table 9, which are mainly influenced by the coefficient of restitution, are compared to simulations applying the same properties (comp. Table 8). They are initially obtained with the DEM parameters determined in section 3 and thereafter, with the best fit of the adjustment.

a

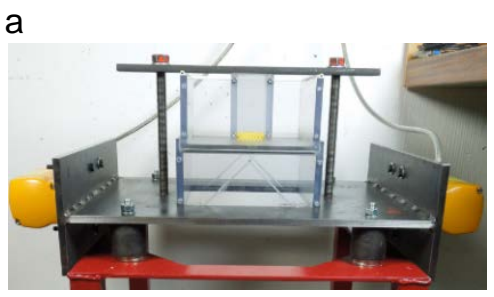

b

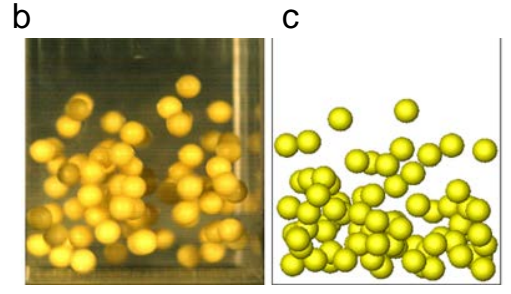

d

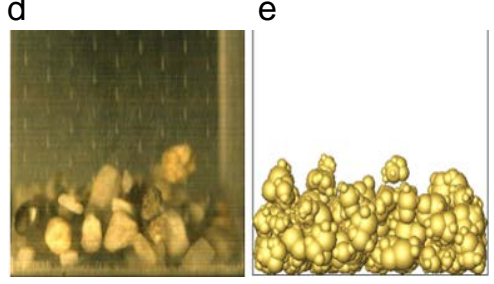

Fig. 11: (a) Experimental set-up to measure the bed height on a vibrating plate as well as resulting particle beds for a filling of one layer of (b,c) $5 \mathrm{~mm}$ POM spheres and (d,e) gravel in (b,d) the experiments and (c,e) the simulations.

371 Applying the initial DEM parameters, the results reveal some good agreements between

372 simulations and experiments but also deviations up to $17 \%$ between the "Top" values in some

373 cases. The "Bottom" values are mostly lower in the simulations than in the experiments but the

374 absolute deviations are less than one respective particle in all cases. After the adjustment, the

375 deviations of the "Top" values are minimized significantly (all below $10 \%$ ), whereas the

376 "Bottom" values in the simulations are only slightly adjusted and still reveal a few deviations. 
Table 9: Experimental results of measuring the dynamic bed height on a vibrating plate and comparison to simulations applying DEM parameters determined in section 3 as well as after the adjustment.

\begin{tabular}{l|cccc|c}
\multicolumn{1}{c}{ Average bed height [mm] } & \multicolumn{3}{c|}{ POM } & gravel \\
\hline Experiment - 1 Layer - Top & $5 \mathrm{~mm}$ & $7 \mathrm{~mm}$ & $10 \mathrm{~mm}$ & 5 / 7 / 10 mm & 3.15 - 5.6 mm \\
Simulation - 1 Layer - Top & 41.40 & 35.70 & 35.50 & 49.90 & 20.70 \\
Simulation - 1 Layer - Top - adjusted & 39.80 & 42.10 & 38.86 & 43.64 & 21.54 \\
Experiment - 1 Layer - Bottom & 3.20 & 37.31 & 36.87 & 49.64 & 20.88 \\
Simulation - 1 Layer - Bottom & 2.69 & 3.99 & 6.26 & 8.07 & 2.74 \\
Simulation - 1 Layer - Bottom - adjusted & 4.20 & 4.05 & 6.45 & 4.62 & 1.14 \\
Experiment - 20 \% - Top & 35.90 & 37.10 & 46.00 & 40.90 & 29.00 \\
Simulation - 20 \% - Top & 30.85 & 36.55 & 46.46 & 34.12 & 26.40 \\
Simulation - 20 \% - Top - adjusted & 35.77 & 37.29 & 46.44 & 40.38 & 27.50 \\
Experiment - 20 \% - Bottom & 1.39 & 1.82 & 4.07 & 3.46 & 2.44 \\
Simulation - 20 \% - Bottom & 0.74 & 1.24 & 2.50 & 0.87 & 0.80 \\
Simulation - 20 \% - Bottom - adjusted & 1.35 & 1.34 & 2.69 & 1.21 & 0.95 \\
Experiment - 50 \% - Top & 56.20 & 54.10 & 58.60 & 59.30 & 58.22 \\
Simulation - 50 \% - Top & 53.15 & 53.69 & 54.41 & 54.53 & 59.97 \\
Simulation - 50 \% - Top - adjusted & 55.90 & 54.4 & 57.80 & 57.24 & 59.00 \\
Experiment - 50 \% - Bottom & 1.17 & 1.29 & 1.18 & 0.98 & 2.42 \\
Simulation - 50 \% - Bottom & 0.81 & 0.92 & 0.73 & 0.64 & 1.20 \\
Simulation - 50 \% - Bottom - adjusted & 1.90 & 2.10 & 2.50 & 1.93 & 1.23 \\
\hline
\end{tabular}

\section{5. Application of the adjusted DEM parameters for batch-screening}

382 The DEM parameters after the adjustment of section 4 are listed in Table 10. Particularly, the 383 sliding friction coefficients between particles and walls have to be increased by an average 384 factor of 2.68 and 1.96 for acryl and metal, respectively. Additionally, the sliding friction 385 between particles is increased by an average factor of 1.27 whereas the rolling friction and the 386 restitution coefficients are adjusted in both directions. All these parameters are applied for 387 batch-screening of well mixed POM spheres with three different size classes and gravel 388 particles with the particle size distribution outlined in Fig. 2. The particle and experimental 389 properties as outlined in Table 1 and Table 11 are used in the simulations.

390 Table 10: DEM parameters after the adjustment for POM spheres and quartz gravel particles. For gravel the rolling friction is 391 neglected.

\begin{tabular}{c|c|ccc}
\hline neglected. & \multicolumn{5}{c}{} & $\mu_{c}$ & $\mu_{\text {roll }}$ & $e^{n}$ \\
\hline Contact partner 1 & Contact partner 2 & $0.6649 / 0.4074$ & $4.30 \mathrm{E}-05 / 6.99 \mathrm{E}-05$ & $0.8145 / 0.8412$ \\
\hline $5 \mathrm{~mm}$ POM & & $0.4415 / 0.3561$ & $8.66 \mathrm{E}-05 / 6.01 \mathrm{E}-05$ & $0.7531 / 0.8013$ \\
$7 \mathrm{~mm}$ POM & Acryl (side walls) / metal (screen wire) & $0.5330 / 0.2816$ & $5.16 \mathrm{E}-05 / 4.91 \mathrm{E}-05$ & $0.8195 / 0.8055$ \\
$10 \mathrm{~mm}$ POM & & $0.3710 / 0.5461$ & $-/-$ & $0.5334 / 0.3966$ \\
Gravel particle & & $0.2395 / 0.3237 /$ & $3.83 \mathrm{E}-05 / 4.00 \mathrm{E}-05$ & $0.8676 / 0.8686 /$ \\
\hline $5 \mathrm{~mm}$ POM & $5 \mathrm{~mm}$ POM / 7 mm POM / 10 mm POM & 0.2935 & $/ 3.30 \mathrm{E}-05$ & 0.7701 \\
$7 \mathrm{~mm}$ POM & $7 \mathrm{~mm}$ POM / 10 mm POM & $0.4882 / 0.4673$ & $3.89 \mathrm{E}-05 / 8.92 \mathrm{E}-05$ & $0.7303 / 0.8209$ \\
$10 \mathrm{~mm}$ POM & $10 \mathrm{~mm}$ POM & 0.4229 & $3.844 \mathrm{E}-05$ & 0.7653 \\
Gravel particle & Gravel particle & 0.5381 & - & 0.8676 \\
\hline
\end{tabular}


The vibration parameters are obtained like in section 4.4, but here the transient period of the screen motion before $t=0.61 \mathrm{~s}$ is also measured and applied to the simulations as well as the removal of the retaining plate below the apertures which lasts $t=0.1 \pm 0.02 \mathrm{~s}$.

Table 11: Experimental properties for batch-screening experiments.

\begin{tabular}{c|c}
\hline Properties & POM / gravel \\
\hline Floor area $[\mathrm{m}]$ & $0.185 \times 0.185$ \\
Aperture size $[\mathrm{mm}]$ & $8.00 \pm 0.02 / 5.80 \pm 0.01$ and $4.00 \pm 0.01$ \\
Wire Diameter $[\mathrm{mm}]$ & $1.5 \pm 0.01$ \\
Scree wire profile $[-]$ & rectangular \\
Particle mass $[\mathrm{kg}]$ & 1 \\
Amplitude $[\mathrm{mm}]$ & $1.20 \pm 0.04$ \\
Frequency $[\mathrm{Hz}]$ & 44 and 54 \\
Stroke angle $\left[{ }^{\circ}\right]$ & $\sim 90$ \\
\hline
\end{tabular}

Investigations for POM spheres are performed for two different frequencies and the results averaged over a sufficient number of experiments (standard deviation represented by the vertical error bars) and simulations reveal a good agreement with only a few deviations between simulations and experiments (comp. Fig. 12a). The horizontal error bars represent the response time of the balance. The average deviations are for $44 \mathrm{~Hz} 0.0164$ and for $54 \mathrm{~Hz}$ 0.0083. In case of quartz gravel, a frequency of $54 \mathrm{~Hz}$ and two different mesh sizes are applied resulting in a fast particle depletion for the aperture size of $5.8 \mathrm{~mm}$ and in a low passage rate for the aperture size of $4 \mathrm{~mm}$.
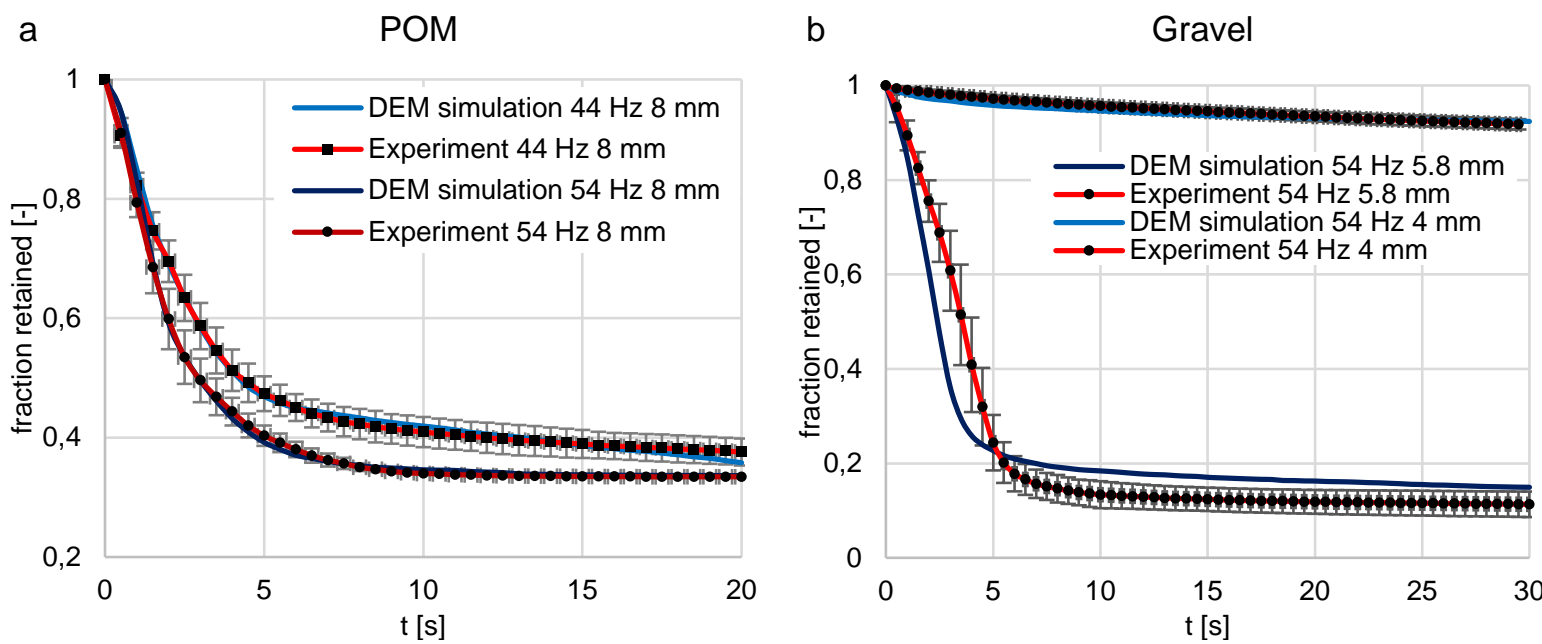

406

407

408

409

410

411

412

413

Fig. 12: Fraction retained on the screen over time applying (a) POM spheres for two different frequencies with a mesh size of $8 \mathrm{~mm}$ and (b) gravel for one frequency with mesh sizes of $5.8 \mathrm{~mm}$ and $4 \mathrm{~mm}$ all obtained by experimental investigations (results are averaged over 10 experiments) and DEM simulations, respectively.

The results for the latter also reveal low deviations (average: 0.0074). In contrast, the results with an aperture size of $5.8 \mathrm{~mm}$ reveal larger deviations (average: 0.0554 ) particularly in the first five seconds, where the particles in the simulations pass faster than in the experiment. Thereafter, the passage rate is reduced and too many particles remain on the screen. Reasons for the deviations can be the rounded shape of the particles increasing the stratification and 
passing possibility, the scaling in the particle size classes based on pixels of images or the low number of different shapes per size class used in the simulations. To overcome these discrepancies will be focused on in further investigations.

\section{Conclusions}

DEM parameters for POM spheres of three different size classes and quartz gravel particles with a realistic size distribution have first been determined by single particle tests and later applied to simulations representing three small bulk experiments. The static and dynamic angle of repose as well as the dynamic bed height are measured and particularly, the measurement of the dynamic angle of repose reveals large deviations between simulations and experiments. Motivated to reduce these discrepancies, the DEM parameters are automatically adjusted with an optimization tool, whereby the deviations between simulations and experiments are minimized significantly. The deviations averaged over all bulk tests are reduced from $9.59 \%$ to $2.15 \%$ for the static angle of repose, from $22.19 \%$ to $3.18 \%$ for the dynamic angle of repose and from $7.25 \%$ to $4.15 \%$ for the dynamic bed height (only the "Top" value). The adjusted DEM parameters are than applied to batch-screening simulations and reveal a good agreement with respective experiments in case of POM spheres and for quartz gravel with a low passage rate. In contrast, in case of the quartz gravel particles applying a large aperture size, the simulation overpredicts the passage rate in the first five seconds but more particles remain on the screen afterwards which indicates that the screening simulation needs further improvement. Investigations with another less rounded particle shape are currently ongoing and more different shapes will be applied for each size class. Nevertheless, it could be shown that the results of single particle tests can only be used as initial values for DEM parameters which have to be further adjusted to minimize deviations between simulations and bulk experiments. After an appropriate automatic adjustment of the DEM parameters, the results of bulk experiments applying spherical and non-spherical particles and the results of more complex processes applying spheres are represented well. In further studies, the batchscreening will be integrated in the adjustment process to enhance the accuracy.

\section{Acknowledgements}

442 The authors gratefully acknowledge the support by DFG within project SPP 1679 through grant 443 number KR3446/7-2. The original form of the DEM-code "DEM-Calc" applied is based on a 444 development of LEAT, Ruhr-Universität Bochum, Germany. The code "DEM-Calc" has then been continuously extended both at Ruhr-Universität Bochum and Technische Universität Berlin, Germany. We thank all who have contributed. 


\section{References}

448 [1] Liu, K.: Some factors affecting sieving performance and efficiency. Powder Technol. 193, 208-213 (2009)

450

451

452

453

454

455

456

457

458

459

460

461

462

463

464

465

466

467

468

469

470

471

472

473

474

475

476

477

478

479

480

481

482

483

484

485

486

487

488

489

490

[2] Grozubinsky, V., Sultanovitch, E., Lin, I.J.: Efficiency of solid particle screening as a function of screen slot size, particle size, and duration of screening - The theoretical approach. Int. J. Miner. Process. 52, 261-272 (1998)

[3] Cundall, P.A., Strack, O.D.: A discrete numerical model for granular assemblies. Geotechnique 29, 47-65 (1979)

[4] Cleary, P.W., Sinnott, M.D., Morrison, R.D.: Separation performance of double deck banana screens - Part 1: Flow and separation for different accelerations. Miner. Eng. 22, 1218-1229 (2009)

[5] Cleary, P.W., Sinnott, M.D., Morrison, R.D.: Separation performance of double deck banana screens - Part 2: Quantitative predictions. Miner. Eng. 22, 1230-1244 (2009)

[6] Delaney, G.W., Cleary, P.W., Hilden, M., Morrison, R.D.: Testing the validity of the spherical DEM model in simulating real granular screening processes. Chem. Eng. Sci. 68, 215-226 (2012)

[7] Kruggel-Emden, H., Elskamp, F.: Modeling of Screening Processes with the Discrete Element Method Involving Non-Spherical Particles. Chem. Eng. Technol. 37, 847-856 (2014)

[8] Yoshida, Y., Ishikawa, S., Shimosaka, A., Shirakawa, Y., Hidaka, J.: Estimation Equation for Sieving Rate Based on the Model for Undersized Particles Passing through Vibrated Particle Bed. J. Chem. Eng. Japan 46, 116-126 (2013)

[9] Li, Y., Xu, Y., Thornton, C.: A comparison of discrete element simulations and experiments for "sandpiles" composed of spherical particles. Powder Technol. 160, 219-228 (2005)

[10] Grima, A.P., Wypych, P.W.: Investigation into calibration of discrete element model parameters for scale-up and validation of particle-structure interactions under impact conditions. Powder Technol. 212, 198-209 (2011)

[11] Chen, H., Liu, Y.L., Zhao, X.Q., Xiao, Y.G., Liu, Y.: Numerical investigation on angle of repose and force network from granular pile in variable gravitational environments. Powder Technol. 283, 607-617 (2015)

[12] Coetzee, C.J., Els, D.N.J.: Calibration of granular material parameters for DEM modelling and numerical verification by blade - granular material interaction. $\mathrm{J}$. Terramechanics 46, 15-26 (2009)

[13] Natsui, S., Ueda, S., Oikawa, M., Fan, Z., Kano, J., Inoue, R., Ariyama, T.: Optimization of Physical Parameters of Discrete Element Method for Blast Furnace and Its Application to the Analysis on Solid. ISIJ Int. 49, 1308-1315 (2009)

[14] Alonso-Marroquín, F., Ramírez-Gómez, Á., González-Montellano, C., Balaam, N., Hanaor, D.A.H., Flores-Johnson, E.A., Gan, Y., Chen, S., Shen, L.: Experimental and numerical determination of mechanical properties of polygonal wood particles and their flow analysis in silos. Granul. Matter 15, 811-826 (2013)

[15] Barrios, G.K.P., de Carvalho, R.M., Kwade, A., Tavares, L.M.: Contact parameter estimation for DEM simulation of iron ore pellet handling. Powder Technol. 248, 84-93 (2013) 
[16] Horabik, J., Molenda, M.: ScienceDirect Parameters and contact models for DEM simulations of agricultural granular materials: A review. Biosyst. Eng. 147, 206-225 (2016)

[17] Coetzee, C.J.: Calibration of the discrete element method and the effect of particle shape. Powder Technol. 297, 50-70 (2016)

[18] Dobrohotoff, P.B., Imranullah, S., Maggi, F., Alonso-Marroquin, F.: Optimal description of two-dimensional complex-shaped objects using spheropolygons. Granul. Matter 14, 651-658 (2012)

[19] Höhner, D., Wirtz, S., Scherer, V.: A numerical study on the influence of particle shape on hopper discharge within the polyhedral and multi-sphere discrete element method. Powder Technol. 226, 16-28 (2012)

[20] Höhner, D., Wirtz, S., Scherer, V.: A study on the influence of particle shape on the mechanical interactions of granular media in a hopper using the Discrete Element Method. Powder Technol. 278, 286-305 (2015)

[21] Li, C.-Q., Xu, W.-J., Meng, Q.-S.: Multi-sphere approximation of real particles for DEM simulation based on a modi fi ed greedy heuristic algorithm. Powder Technol. 286, 478487 (2015)

[22] Benvenuti, L., Kloss, C., Pirker, S.: Identification of DEM simulation parameters by Artificial Neural Networks and bulk experiments. Powder Technol. 291, 456-465 (2016)

[23] Zhu, H.P., Zhou, Z.Y., Yang, R.Y., Yu, A.B.: Discrete particle simulation of particulate systems: Theoretical developments. Chem. Eng. Sci. 62, 3378-3396 (2007)

[24] Zhu, H.P., Zhou, Z.Y., Yang, R.Y., Yu, A.B.: Discrete particle simulation of particulate systems: A review of major applications and findings. Chem. Eng. Sci. 63, 5728-5770 (2008)

[25] Munjiza, A., Latham, J.P., John, N.W.M.: 3D dynamics of discrete element systems comprising irregular discrete elements - integration solution for finite rotations in 3D. Int. J. Numer. Methods Eng. 56, 35-55 (2003)

[26] Kruggel-Emden, H., Rickelt, S., Wirtz, S., Scherer, V.: A study on the validity of the multisphere Discrete Element Method. Powder Technol. 188, 153-165 (2008)

[27] Höhner, D., Wirtz, S., Kruggel-Emden, H., Scherer, V.: Comparison of the multi-sphere and polyhedral approach to simulate non-spherical particles within the discrete element method: Influence on temporal force evolution for multiple contacts. Powder Technol. 208, 643-656 (2011)

[28] Kruggel-Emden, H., Kačianauskas, R.: Discrete element analysis of experiments on mixing and bulk transport of wood pellets on a forward acting grate in discontinuous operation. Chem. Eng. Sci. 92, 105-117 (2013)

[29] Kruggel-Emden, H., Sudbrock, F., Wirtz, S., Scherer, V.: Experimental and numerical investigation of the bulk behavior of wood pellets on a model type grate. Granul. Matter 14, 681-693 (2012)

[30] Kruggel-Emden, H., Simsek, E., Rickelt, S., Wirtz, S., Scherer, V.: Review and extension of normal force models for the Discrete Element Method. Powder Technol. 171, 157173 (2007)

[31] Kruggel-Emden, H., Wirtz, S., Scherer, V.: A study on tangential force laws applicable to the discrete element method (DEM) for materials with viscoelastic or plastic behavior. Chem. Eng. Sci. 63, 1523-1541 (2008) 
[32] Renzo, A. Di, Maio, F.P. Di: An improved integral non-linear model for the contact of particles in distinct element simulations. Chem. Eng. Sci. 60, 1303-1312 (2005)

538 [33] Cleary, P.W.: Large scale industrial DEM modelling. Eng. Comput. 21, 169-204 (2004)

539 [34] Kruggel-Emden, H., Stepanek, F., Munjiza, A.: A comparative study of reaction models 540 applied for chemical looping combustion. Chem. Eng. Res. Des. 89, 2714-2727 (2011)

541

542

[35] Zhou, Y.C., Wright, B.D., Yang, R.Y., Xu, B.H., Yu, A.B.: Rolling friction in the dynamic

543

544

545 simulation of sandpile formation. Physica A 269, 536-553 (1999)

[36] Sudbrock, F., Simsek, E., Rickelt, S., Wirtz, S., Scherer, V.: Discrete element analysis of experiments on mixing and stoking of monodisperse spheres on a grate. Powder Technol. 208, 111-120 (2011)

546

547

[37] Goldberg, D.E.: Genetic Algorithms in Search, Optimization and Machine learning. Addison-Wesley Longman, Boston (1989)

548 